\title{
Propuesta metodológica para la creación de un sistema de información arqueológica de la ciudad
}

\author{
Eva Mª Alcázar Hernández**
}

Uno de los campos que se ha revelado como más complejo y problemático dentro del marco general de la intervención arqueológica en Centros Habitados, es la necesidad de conseguir un sistema de registro, catalogación y almacenamiento de la información extraída a través de las intervenciones arqueológicas, que permita tanto a los arqueólogos investigadores, como a la propia Administración, gestionar, manejar y analizar la enorme cantidad de datos recabados hasta ahora, y los que se recogerán en un futuro.

La empresa es harto complicada. Son numerosísimas las intervenciones arqueológicas realizadas, muy diferentes los criterios metodológicos usados según las ciudades en las que se trabaje, los equipos de investigación o arqueólogos profesionales implicados, y las distintas estrategias adoptadas en cada intervención, en cada yacimiento, dependiendo de cada caso-; la situación se agrava aún más porque las intervenciones arqueológicas en áreas urbanas deben adaptarse a la típica fragmentación en pequeños solares, que dificultan sobremanera el intento de un registro sistemático y globalizador, que a la vez no menoscabe calidad y riqueza de la información.

Sin embargo, y a pesar de la complejidad del problema, es evidente que, para que el gran esfuerzo realizado en la última década para salvaguardar y estudiar el Patrimonio Arqueológico de nuestras ciudades tenga resultados, es necesaria la sistematización de la información: para que los datos sean explotables, es indispensable que exista una estructura de base, que alcance una homogeneidad mínima, constituyéndose en un auténtico útil de investigación y administración.
La Informática (Gardin, 199|), y más en concreto, los programas informáticos denominados S.I.G., Sistemas de Información Geográfica (Bosque, 1992), constituyen el instrumento que responde a las necesidades de la Arqueología Urbana de almacenar, sistematizar, gestionar y analizar la información que proporciona sobre la Historia de la Ciudad, posibilitándonos la creación de nuestro propio Sistema de Información Arqueológica (S.I.A.), entendido como extensión de los S.I.G., adaptado a los imperativos de la Arqueología.

El desarrollo de una estructura informática abierta, que permita la construcción de un registro de la información arqueológica, para su posterior manejo y análisis, es fundamental, tanto para la Administración como para los investigadores, en orden a mejorar la conservación y el conocimiento de esta importantísima parte de nuestra herencia cultural.

Son diversas las experiencias que, en este sentido, se están realizando en Europa. El Sistema ArcheoDATA, desarrollado en la île de Francia (Arroyo-Bishop, 1991) es una propuesta interesante, de cara a la actividad futura. El Dipartimento di Archeologia e Storia delle Arti de la Università degli Studi de Siena, está, igualmente utilizando un Programa S.I.G., para construir la Carta Arqueológica de la Provincia de Siena. En la Península, Aragón es pionero en la configuración de una Base de Datos y Ficha Informatizada de registro, para el Proyecto "Carta Arqueológica de Aragón".

Estas "implementaciones", una vez estudiadas en profundidad, aportan interesantísimas ideas, aunque 
si bien no están configuradas, en sentido estricto, para responder a las necesidades específicas de la Arqueología Urbana. Y esto es un problema esencial: el ordenador y los programas comerciales de S.I.G. son instrumentos valiosos, pero antes de usarlos, hay que tener bien claros los objetivos que se pretenden perseguir, las preguntas que se quieren responder, $y$, sobretodo, se debe tener bien presente, en el caso que nos ocupa, la especificidad de un Registro de Información Arqueológica Urbana, que es reflejo de la problemática de la Arqueología en los centros habitados:

En la ciudad, la intervención más común es la excavación de urgencia, realizada ante la inminencia de una sustitución arquitectónica total o de una reestructuración en profundidad, efectuada en un tiempo breve; la superficie de intervención suele ser el solar, que sólo permite realizar, en la mayoría de los casos, sondeos de pequeño tamaño; el método más comúnmente utilizado es el estratigráfico, que debe superar las dificultades que al análisis opone la típica pluriestratigrafía de las ciudades, afectada por innumerables intervenciones urbanísticas en profundidad, realizadas sobre todo a partir de la Baja Edad Media y hasta nuestros días, que dificultan enormemente, en muchas ocasiones, la interpretación de los estratos más antiguos, o, simplemente, los destruyen; la continua reutilización de estructuras hace necesaria la utilización de otra metodología arqueológica afín, como es el análisis estratigráfico murario; fundamental para entender la topografía de la ciudad en cada momento histórico es el señalar los cambios en los niveles de la base geológica, así como los niveles de aparición de la capa freática, etc.

Por otro lado, los tiempos de estudios de los materiales aparecidos en la excavación suelen ser breves, apresurados; las consultas de documentación bibliográfica, escrita o planimétrica referente al solar excavado, también suelen ser poco exhaustivas; después de realizar el trabajo y dar un diagnóstico, el único canal de difusión de los datos obtenidos es, en general, un Informe, que, sorprendentemente, se considera que "si breve, dos veces bueno"...

A la hora de realizar un registro sistemático, global, y exhaustivo, debemos tener en cuenta, en fin, que el arqueólogo se enfrenta, en cada intervención, con una casuística que no tiene nada de homogénea ni de sistemática, y que no ofrece, en el 95\% de los casos, una interpretación fácil, muchas veces por fragmentaria: Desde la esterilidad arqueológica del terreno por no haber sido habitado hasta el presente siglo o bien por la destrucción de cualquier rastro de estratigrafía por fosas de cimentación recientes, al aprovechamiento en Edad Moderna y Contemporánea de estructuras medievales que descansan a su vez, en niveles de enterramiento ibero-romanos, son innumerables las situaciones que el arqueólogo encuentra y ante las cuales debe reaccionar según su criterio (Salvatierra, en prensa).

Todo este complejo entramado de factores determinantes y configuradores de la Arqueología Urbana y de su práctica fueron los que tuvimos que tener muy presentes a la hora de iniciar nuestro proyecto:

Configurar un modelo de Ficha Informatizada de Registro Arqueológico, matriz de una Base de Datos, cuyo objetivo último y globalizador era el de inventariar, almacenar y registrar en ella todo el conocimiento posible sobre el pasado histórico de una ciudad.

La unidad básica de la ficha es el Solar, construido o no, al ser éste la superficie habitual de intervención. A la ficha se le exige que extraiga absolutamente, toda la información -arqueológica, geográfica, arquitectónica, administrativa- existente sobre el solar.

La información global extraída de todos los solares sometidos a estudio, configurarán la Base de Datos, que tendrá la misión de ayudarnos a comprender la Historia de la Ciudad, una vez que conozcamos y recopilemos todas las evidencias sobre la evolución cronológica del conjunto de solares que conforman el entramado actual de calles $y$, en definitiva, la Ciudad.

Todo ello nos conducirá, pensamos, y en primer lugar, a estar en condiciones de formular hipótesis interpretativas sobre cada período histórico vivido en y por la Ciudad.

En segundo lugar, a través de la Base de Datos, se intentaría cuantificar la pérdida de información producida por la alteración o destrucción del substrato arqueológico, que se ha detectado en la ciudad hasta la fecha, así como la esterilidad arqueológica 
de determinadas zonas. Todo ello, contrastado con la potencia arqueológica hallada en otros sectores del centro habitado, a través de sondeos, nos daría el porcentaje aproximado de la documentación perdida de forma global y zonal, así como por etapas históricas. Igualmente, podríamos delimitar con cierta precisión los sectores que sólo recientemente han sido habitados. Creemos que esta posibilidad es fundamental a la hora de optimizar los recursos de que disponemos y de diseñar futuras estrategias de intervención e investigación en el casco histórico de nuestras ciudades.

La información histórico-arqueológica que de cada solar pretendemos recopilar en la Base de Datos, no es sólo aquella que nos ofrece el subsuelo. Creemos fundamental el recoger, igualmente, la información ofrecida por las estructuras arquitectónicas emergentes, de cualquier época, ya sean monumentales o no, estén catalogadas B.I.C. - incoadas para ello- o no. Preocupándonos de que quede constancia, siempre que sea posible, de las reestructuraciones, restauraciones, o rehabilitaciones de las que han sido objeto, y del estado en que se hallan actualmente los inmuebles edificados en cada solar, el uso que ahora tienen, y los anteriores conocidos.

Para comprobar la efectividad de nuestro proyecto de Sistema de Registro, estamos aplicando la Ficha Informatizada en un prototipo o modelo experimental, para crear una Base de Datos básica, así como para, una vez enfrentada con situaciones reales, efectuar ulteriores correcciones en su diseño, que nos permita un óptimo rendimiento.

Hemos seleccionado diferentes calles de la ciudad de Jaén -nuestro "campo de pruebas"-, en total son cuatro calles y cuatro plazas, todas ellas dentro del casco histórico, desarrollando una exhaustiva y sistemática búsqueda de información sobre cada uno de los solares -construidos o noque conforman dichas calles (en total son 240 solares).

La suerte corrida por estos solares y, en general, por las calles que dibujan, por lo que respecta a su alineación original, a la supervivencia de la arquitectura tradicional y a la conservación de edificios monumentales e históricos, es muy diversa. El proceso de sustitución constructiva ha sido intensísimo en algunas, hasta el punto de no quedar apenas edificios de más de 20 años; en otras, aún quedan importantes islotes arquitectónicos que conservan su originalidad y la alineación primitiva. Hemos tenido en cuenta también, como factor de selección, el que en algunas de estas calles se ubican edificios declarados B.I.C. o incoados para ello, o edificios de interés artístico o histórico. Por supuesto, en casi todas estas calles se han realizado, desde 1987 , numerosas intervenciones arqueológicas con resultados diversos, tanto en lo que respecta a la potencialidad de su estratigrafía, como a las fases culturales detectadas. De esta forma, pensamos, hemos conseguido reunir un amplio espectro en el que se recogen prácticamente todas las situaciones reales a las que se puede enfrentar el investigador cuando estudia el Patrimonio Arqueológico y Arquitectónico de su ciudad: diferentes estados de conservación de los edificios, diferente potencialidad arqueológica, diferente grado de destrucción o sustitución, etc.

Uno de los objetivos inmediatos, era comprobar si la parte de la Ficha dedicada a la información arqueológica, nos permitía crear un registro en el que se pudieran homologar todos los datos recabados en las intervenciones arqueológicas, superando las diferencias en los niveles de información logrados, diferencias que son consecuencia, como he apuntado antes, tanto de la diferente potencialidad arqueológica de cada solar como de la diversa casuística con la que se enfrenta el arqueólogo en cada excavación. Esta fase del trabajo se ha revelado como la más compleja y problemática, como cabía esperar. El reunir toda la documentación aportada por las numerosas intervenciones realizadas en las calles que conforman el prototipo, es una tarea ardua. No bastan los informes redactados por cada arqueólogo, sino que, para que la base de datos alcance un óptimo nivel de rendimiento, necesitamos los diarios de excavación, la planimetría efectuada y los estudios de materiales, siendo su recopilación lenta y dificultosa, tanto por su número como por el tiempo pasado entre cada intervención arqueológica y por los numerosos arqueólogos profesionales implicados. A esto se une el problema de base: la homologación de la información en una ficha única, que no menoscabe calidad al producto final. Tras varios ensayos estamos logrando adecuar y flexibilizar la ficha informatizada de registro que diseñamos inicialmente, una 
vez que hemos comenzado a usarla con las informaciones obtenidas en las intervenciones arqueológicas realizadas en estas calles, que, como he dicho al principio, son de muy diversa índole: desde la que ofrece una estratigrafía prácticamente completa desde poca ibero-romana a nuestros días, hasta aquella en la que la base geológica aparece inmediatamente por debajo del nivel actual de calle, sin que se detecten más restos arqueológicos que los del edificio que acaba de ser demolido, pasando por algunos lamentables casos en los que la obra comenzó antes de que el arqueólogo pudiera ofrecer su diagnóstico, por lo que éste sólo encontró una enorme placa de cemento cimentador.

A esta actividad se ha acompañado la recopilación de información en el Archivo del Excelentísimo Ayuntamiento de Jaén, sobre todo para conocer los momentos en los que se abrieron las calles, consultando las Actas Capitulares, y en los que habían sido sustituidos los edificios. En este sentido, las Licencias de Obras Mayores concedidas por el Ayuntamiento, se han revelado como una fuente fundamental, pues, además de permitirnos saber la fecha de construcción de los inmuebles, así como la de la demolición de los originales, especifica el tipo de construcción, el uso al que se destina, el arquitecto responsable, y la existencia, en las nuevas construcciones, de elementos que hayan podido destruir potencialidad arqueológica: sótanos, aparcamientos subterráneos, etc. Las Licencias de Obras Menores, también son muy útiles para conocer qué modificaciones han sufrido los edificios originales con el paso del tiempo.

Junto a esto, intentamos rastrear los cambios de alineaciones en las calles, las periódicas renovaciones de la red de saneamiento pública, las intervenciones de la Compañía Telefónica, etc... En definitiva, todas aquellas intervenciones en el subsuelo que hayan podido alterar la estratigrafía.

Igualmente, estamos llevando a cabo un vaciado de todas las revistas locales editadas en Jaén desde el siglo pasado, que se interesaran por temas de Arqueología y Arquitectura, para recabar noticias sobre posibles hallazgos arqueológicos, así como para conocer la historia de los edificios más señeros de estas calles. Hemos entrado en contacto también con arquitectos que se hayan interesado por la historia contemporánea del urbanismo en Jaén, así como con historiadores del Arte que nos puedan documentar sobre los tipos de construcción tradicionales en Jaén.

Fundamental sería, a este respecto, la posibilidad de acceder a los archivos de la Consejería de Cultura, en especial al de la Comisión de Patrimonio Histórico Andaluz, para estudiar los expedientes creados por ella.

La investigación en archivos y bibliotecas la estamos completando con prospecciones sistemáticas de las calles, en las cuales inventariamos uno a uno los inmuebles edificados en cada solar, cotejando las informaciones recogidas con la realidad. A la información global de todos los solares, se acompaña un álbum de fotografías en las que se reflejan el estado actual de los edificios.

Nuestro objetivo fundamental en los próximos meses será la de completar las informaciones recogidas en la Base de Datos, continuando con las actividades descritas más arriba, para, a continuación poner en ejecución, a partir de la Base de Datos creada, un Sistema de Información Arqueológica Urbana.

La imbricación de nuestra Base de Datos en un S.I.G., abre excelentes posibilidades. Al ser todos los datos georreferenciables, tendremos en cualquier momento la plasmación cartográfica y planimétrica de la información, creciendo infinitamente las posibilidades de gestión e investigación científica de los datos aportados por los solares urbanos.

La implementación de un Sistema de Información Arqueológica ofrece, además, la ventaja y posibilidad de añadir ficheros de información mucho más especializados y no necesariamente georreferenciables, sobre diferentes temas: estudio de los materiales hallados, vegetación autóctona, demografía, documentación de archivo y bibliográfca, etc., que irán completando la Base de Datos inicial, interrelacionándose con ella.

El primer paso a ultimar es la digitalización del plano de las calles que conforman nuestro modelo o prototipo, solar por solar, a la espera de finalizar con la recogida de datos, para tener así el soporte cartográfico básico de la información. Un segundo paso es digitalizar, dentro de cada solar excavado, 
las estructuras aparecidas de épocas anteriores, introduciéndose también las fotografías de los edificios que ahora lo ocupan, y que ya han sido realizadas.

Seguidamente, se introducirá nuestra Base de datos, realizada en DBASE IV, en un programa comercial S.I.G.

Las posibilidades que se abren para la conservación, catalogación y estudio del Patrimonio Arqueológico y Arquitectónico urbano son inmensas.

Ni qué decir tiene que, una de las consecuencias más directas de la construcción de este Sistema de Información Arqueológica Urbana, es la posibilidad de fabricar una Carta Arqueológica, y además -y sobretodo, ante la urgencia del problema- una Carta de Riesgo (W.AA., 1992). Efectivamente, a partir de las informaciones temáticas que conforman nuestra Base de Datos, que realiza una precisa catalogación de la presencia y distribución dentro de la trama urbana del Patrimonio Arqueológico y Arquitectónico, así como una puntual recogida de todas las informaciones relativas a su estado de conservación o deterioro, y a los factores de peligrosidad que lo amenazan, se puede establecer un sistema de correlaciones para determinar las con- cretas relaciones causales y de probabilidad que existen entre el Patrimonio Arqueológico, Arquitectónico e Histórico-Artístico de la ciudad, su estado de conservación y las concretas circunstancias y factores que provocan su destrucción y deterioro. Pudiéndose medir su auténtica vulnerabilidad, a partir de lo cual diseñar estrategias para su conservación, restauración, estudio, etc.

\section{BIBLIOGRAFÍA}

ARROYO-BISHOP, D. (199I): "El Sistema ArchoDATA: hacia la creación de un Sistema de Información Arqueológica" en Aplicaciones Informáticas en Arqueología, Complutum N N I. Madrid, pp. I67-174.

BOSQUE SENDRA, J. (1992): Sistemas de Información Geográfica. Madrid.

GARDIN, J.C. (|99|): "La inteligencia artificial en Arqueología: hoy y mañana" en Aplicaciones Informáticas en Arqueología, Complutum, № I. Madrid, pp. $31-40$.

SALVATIERRA, V. (en prensa): "El Patrimonio Arqueológico Urbano". Curso sobre Conservación del Patrimonio Arqueológico. Sevilla.

W.AA. (1992): La Carta de Riesgo. Sevilla. 


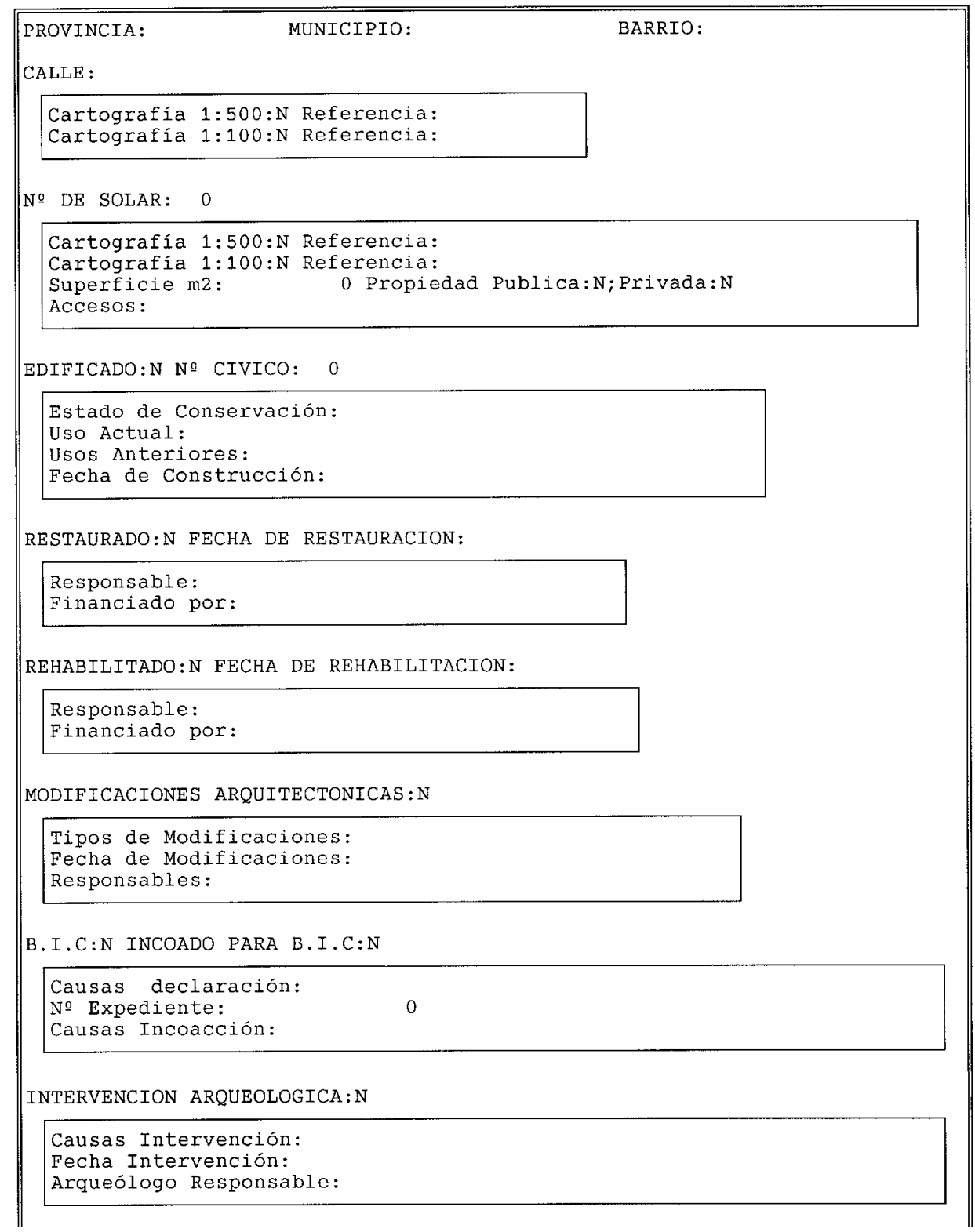

Fig. Modelo de Ficha Informatizada de Registro Arqueológico 


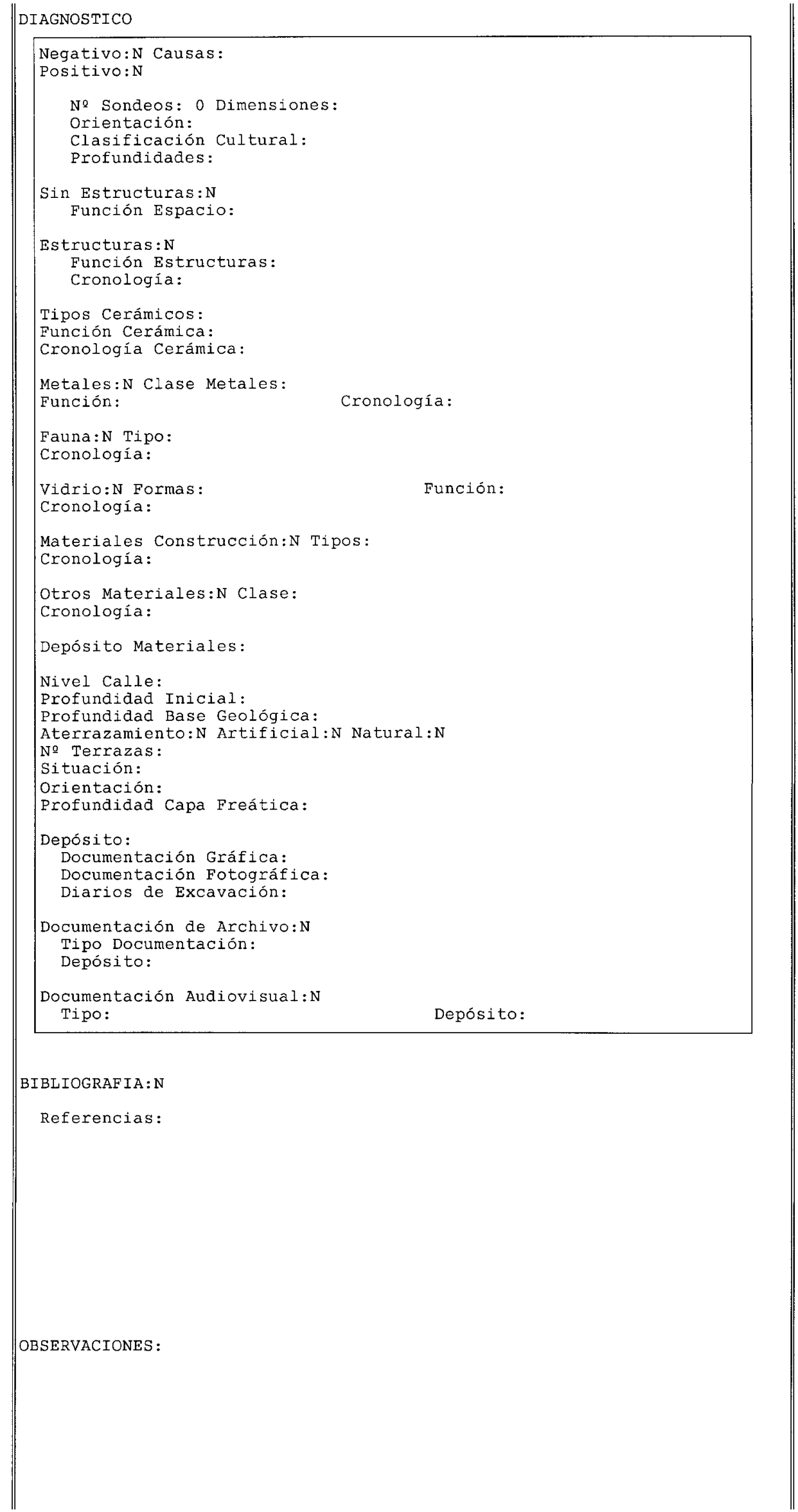

